\title{
Oval Window Niche Height: Quantitative Evaluation with CT before Stapes Surgery for Otosclerosis
}

\author{
E. Ukkola-Pons, D. Ayache, Y. Pons, M. Ratajczak, C. Nioche, and M. Williams
}

\begin{abstract}
BACKGROUND AND PURPOSE: Stapes surgery for otosclerosis can be challenging when the oval window niche is narrow. We analyzed the reliability of CT to evaluate the height of the OWN and propose a quantitative criterion to distinguish normal and narrow OWNs.

MATERIALS AND METHODS: Fifty-six patients were scheduled for primary stapes surgery and, with available preoperative CT scans, were prospectively enrolled in the study at a tertiary care hospital. OWN height was measured on coronal CT and qualitatively evaluated during surgery. CT findings and surgical observations were matched to determine the preoperative imaging criterion of a narrow OWN.

RESULTS: OWN was found to be narrow during surgery in 8 of 56 patients (14\%). On CT, mean OWN height measurement was $1.1 \mathrm{~mm}$ for the narrow group and $1.8 \mathrm{~mm}$ for the normal OWN surgical cases. The cutoff between normal and narrow OWN was computed at $1.3 \mathrm{~mm}$ by using discriminant analysis and at $1.4 \mathrm{~mm}$ with boxplot analysis. These CT cutoff values allowed a correct classification of "normal" and "narrow" OWN, compared with visual evaluation during surgery.
\end{abstract}

CONCLUSIONS: Measurements of the OWN height provide an accurate and relevant evaluation of this region before otosclerosis surgery. A width below $1.4 \mathrm{~mm}$ should be considered at risk for technical difficulties during the stapes footplate approach.

ABBREVIATIONS: $L D A=$ linear discriminant analysis; $M P R=$ multiplanar reconstruction; $O W N=$ oval window niche

$\mathbf{P}$ reoperative CT may be useful when surgical treatment of otosclerosis is planned. ${ }^{1-5} \mathrm{CT}$ confirms the diagnosis in most cases, allows detection of diseases mimicking otosclerosis or associated middle ear abnormalities that can lead to postoperative failures, and is also able to depict labyrinthine anomalies that may lead to postoperative sensorineural complications. Moreover, CT allows a preoperative evaluation of middle ear anatomy, particularly the region of the oval window and the tympanic segment of the fallopian canal. Assessment of this region is essential because a narrow oval window niche may be responsible for technical difficulties during the procedure.

OWN height is usually qualitatively evaluated on coronal CT scans, but this method of evaluation is based only on the expertise of the viewer in the field of ear imaging and therefore is subject to interobserver variations. A quantitative method, based on mea-

Received June 4, 2012; accepted after revision August 1.

From the Departments of Medical Imaging (E.U.-P.) and Ear, Nose, and Throat (Y.P., C.N.), l'Hôpital duVal de Grâce, Paris, France; and Departments of Ear, Nose, and Throat (D.A., M.R.) and Medical Imaging (M.W.), Fondation Ophtalmologique A. de Rothschild, Paris, France.

Please address correspondence to E. Ukkola-Pons, MD, Department of Medical Imaging, l'Hôpital Val de Grâce, 74 Bd Port Royal, 75230 Paris Cedex 5, France; e-mail:yoann.pons@wanadoo.fr

http://dx.doi.org/10.3174/ajnr.A3354 surement of the OWN height, by using precise and reproducible anatomic landmarks, could be considered more accurate.

The purpose of this study was to assess the clinical relevance of precise measurement of the OWN compared with intraoperative visual evaluation by an expert ear, nose, and throat surgeon (D.A.) and to determine the threshold between normal and narrow oval windows.

\section{MATERIALS AND METHODS}

A prospective single-center study was performed from May 2010 to June 2011. Our series consisted of 56 patients with otosclerosis, scheduled for surgery and having a CT examination available for measurement. In our institution, CT is routinely performed when stapes surgery for otosclerosis is being considered. The sex ratio was female/male, 4:1; the mean age was 45 years (range, 18-61 years).

Two senior radiologists (E.U.-P. and M.W.), blinded to the surgical findings, analyzed routine preoperative CT examinations of both ears (112 temporal bones), performed with a highresolution bone-reconstruction algorithm on a 16-row CT unit (Sensation 16; Siemens, Erlangen, Germany). In all the patients, the OWN height was measured on coronal multiplanar reconstructions perpendicular to the plane of the lateral semicircular canal (helical axial 0.6- $\mathrm{mm}$ acquisition; increment, $0.3 \mathrm{~mm}$ ). 

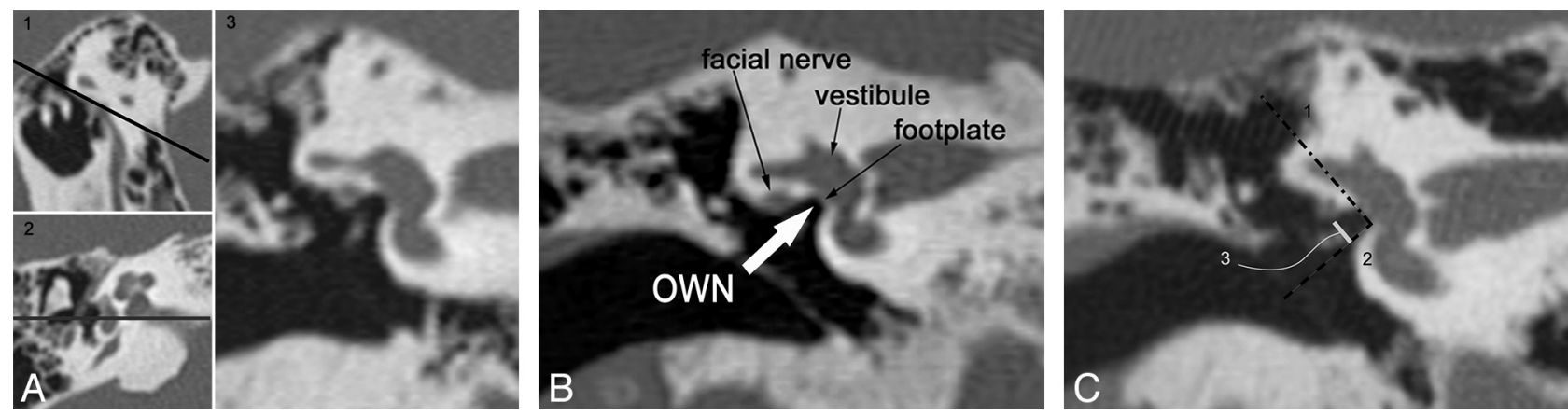

FIG 1. Measurement of the OWN height in a normal OWN case. A, Parallel line (black) to the lateral semicircular canal in a sagittal plane (1) allows obtaining the axial plane (2); a line (gray) is drawn through the middle of the footplate stapes to obtain the straight coronal plane to perform the OWN height measurement (3). B, CT anatomic landmarks of the coronal MPR going through the middle of the stapes footplate. C, Measurement technique. A first line $(1$, dash-dot) is drawn parallel to the main vestibule axis; then a perpendicular line $(2$, dash) is drawn through the inferior margin of the oval window. Eventually, a line (3, solid) perpendicular to the former is drawn between the line (dash) and the inferior aspect of the facial nerve; this third line corresponds to the OWN height, measured here at $1.9 \mathrm{~mm}$.



FIG 2. Boxplot of data from OWN height measurements when the OWN was described as normal (left side) and as narrow (right side).

The measurement of the OWN height was performed on coronal views going through the middle part of the stapes footplate. First, a line (dash-dot) was drawn along the main vestibule axis; then, a second line (dash) perpendicular to the prior line was drawn through the inferior part of the oval window. Eventually, observers measured the distance (solid line) between the second line and the inferior aspect of the facial nerve canal, considered the OWN height (Fig $1 A-C$ ).

For each patient, CT measurements were compared with intraoperative evaluation of the OWN height as reported by the expert otologic surgeon (D.A.). The OWN was considered narrow on the basis of both visual evaluation by the surgeon and difficulty introducing an 0.8-mm footplate perforator. Each CT scan was preoperatively analyzed by the surgeon, who was not aware of the radiologist's measurements or comments about the OWN height and who did not perform OWN height measurements.

CT scans were also assessed for other causes of a narrow oval window such as an overhanging facial nerve or a hypertrophic otosclerotic focus located on the inferior wall of the OWN.

Statistical analysis was performed by using Student $t$ tests, boxplots, and linear discriminant analysis by a "leave-one-out"

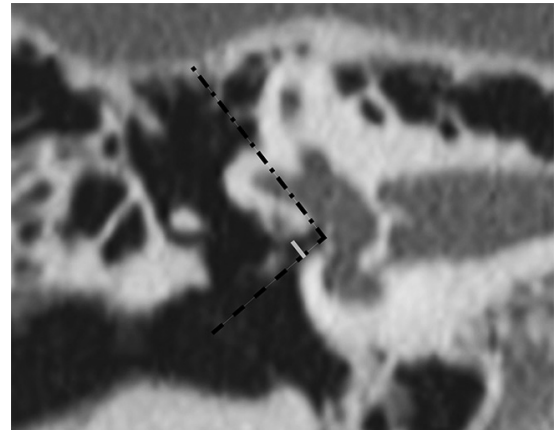

FIG 3. Narrow OWN with hypertrophic bony walls, measured at $0.9 \mathrm{~mm}$ (solid line) on coronal MPR.

method, to determine a threshold between normal and narrow OWNs.

\section{RESULTS}

The mean OWN height measurement was $1.67 \mathrm{~mm}$. In 8 patients (14\%), the OWN was considered narrow during the surgical procedure, with a mean OWN height of $1.1 \mathrm{~mm}$ (range, $0.8-1.4 \mathrm{~mm}$ ); difficulties during the footplate approach were encountered in 5 of these 8 patients. OWN narrowing was due to hypertrophic otosclerotic bony walls in 5 patients and to an overhanging facial canal or nerve in 3 patients (prolapse of the fallopian bony canal in 2 patients, dehiscence of the bony canal with an overhanging facial nerve in 1 patient). Despite narrowing of the $\mathrm{OWN}$, the procedure was completed in all the cases. In 2 cases, the inferior edge of the OWN had to be drilled to reach the footplate. In 4 cases, a smaller footplate perforator than is routinely used $(0.6$ versus $0.8 \mathrm{~mm}$ diameter) was needed to create the footplate hole. In the case of a dehiscent overhanging facial nerve, the nerve had to be gently displaced superiorly to improve footplate exposure. In 48 patients $(86 \%)$, the OWN was considered normal during surgery, with a mean OWN height measured at $1.8 \mathrm{~mm}$ (range, 1.4-2.4 $\mathrm{mm}$ ).

A high correlation between CT OWN height measurements and surgical observations was observed with a $P$ value $<0.05$. In all cases in which the surgeon found a narrow OWN, the OWN height measured $<1.4 \mathrm{~mm}$ as described in the boxplot (Fig 2). The cutoff value under which the surgeon described the OWN as

AJNR Am J Neuroradiol 34:1082-85 May 2013 www.ajnr.org

1083 



termined. All patients were investigated by the same expert otologic surgeon, and our measurements were correlated to actual surgical situations.

Narrowing was due to either hypertrophic OWN bony walls or an overhanging facial nerve. Hypertrophic OWN bony walls can be related to otosclerotic remodelling extending to the superior and/or inferior wall or can be congenital. ${ }^{7}$ In this study, it was not possible to determine why bony walls were hypertrophic in some cases. In 3 patients (5\%), OWN was also due to an overhanging facial ca-

G. Dehiscent tympanic segment of the facial nerve overhanging in front of the oval window on coronal MPR $(A)$ and with the OWN height measurement (solid line, $B$ ).

"narrow" was computed at $1.3 \mathrm{~mm}$ with LDA by a "leave-oneout" method. Interobserver concordance of the OWN height measurements on CT was calculated at $\kappa=0.259$ with a Fleiss $\kappa$ test.

\section{DISCUSSION}

The height of the OWN is relevant to surgical treatment of otosclerosis, but specific criteria for OWN height based on CT measurements are not established, to our knowledge.

In our series, the cutoff between normal and narrow OWN height was computed at $1.3 \mathrm{~mm}$ by using an LDA test; boxplot analysis provided a 1.4-mm cutoff (Fig 3). Both cutoff values led to the same final classification and were correlated to the surgeon's evaluation. When the OWN height measures $<1.4 \mathrm{~mm}$, it should be considered narrow, indicating that technical difficulties may be encountered during stapes footplate approach. Although this threshold between normal and abnormal values on CT is close to the normal width of $1.5 \mathrm{~mm}$ measured in situ on dry temporal bone specimens, ${ }^{6}$ these data obtained from 2 different techniques cannot be compared because of the use of different anatomic landmarks.

Reproducibility of OWN measurements, evaluated by the Fleiss $\kappa$ test, was poor. This result may be explained mainly by a lack of spatial resolution of MPRs. Although most CT workstations provide $0.1 \mathrm{~mm}$ (or even less) measurement steps, this value is smaller than the actual spatial resolution of CT images. For example, with a $7 \mathrm{~cm}$ FOV and a $512 \times 512$ image matrix used on our CT unit, theoretic pixel dimensions in the acquisition plane are close to $0.15 \times 0.15 \mathrm{~mm}^{2}$; therefore, a measurement difference of $0.1 \mathrm{~mm}$ between 2 observers has no significance. Moreover, one must consider that in our study, OWN height measurements are performed on MPRs along the z-axis, of which the spatial resolution is physically restricted by the nominal acquisition section thickness of $0.6 \mathrm{~mm}$. This measurement results in a slight blurring of the margins of minute anatomic structures of the temporal bone and probably explains minor differences in positioning of the reference lines used for measurements. However, even if there was no perfect agreement between the 2 observers, this had no influence on the final result: In all the cases, OWN height was classified in the same category ("narrow" or "normal") by both radiologists.

In the present study, CT measurements were compared with qualitative evaluation of the OWN height during surgery because no preoperative standard measurement has been consensually de- nal or facial nerve (Fig 4) in front of the oval window. This pattern was previously reported in $5 \%,{ }^{8} 25 \%,{ }^{9}$ and $32 \% .{ }^{10}$

Stapes surgery is more challenging with a narrow OWN. ${ }^{11}$ In such a case, the options for the surgeon are to abandon the procedure or to modify his or her surgical technique to enhance footplate visualization - for example, by displacing superiorly a prolapsed facial nerve, reducing the size of the promontory aspect of the footplate, or by using smaller surgical instruments or prosthesis diameters. ${ }^{10-12}$ Those modifications of the usual stapes surgery technique require an experienced surgeon and a longer surgical time. In this study, we included primary surgical procedures exclusively, but some patients were already referred to our ear, nose, and throat department by less experienced surgeons after exploratory tympanotomy that was not completed because of the narrowness of the OWN.

\section{CONCLUSIONS}

Measurement of the OWN height is easy to perform on coronal $\mathrm{CT}$ reformations. This technique provides a quantitative evaluation of the oval window region, highly correlated to qualitative assessment by the surgeon during the procedure. OWN height measurement should be considered in the preoperative work-up before surgical treatment of otosclerosis; an OWN height $<1.4 \mathrm{~mm}$ should be considered narrow, with increased risk of technical difficulties during the stapes footplate approach.

\section{REFERENCES}

1. Lagleyre S, Sorrentino T, Calmels MN, et al. Reliability of highresolution CT scan in diagnosis of otosclerosis. Otol Neurotol 2009;30:1152-59

2. Shin YJ, Deguine O, Cognard C, et al. Reliability of CT scan in the diagnosis of conductive hearing loss with normal tympanic membrane [in French]. Rev Laryngol Otol Rhinol (Bord) 2001;122:81-84

3. Ala Eddine C, Williams M, Ayache D. Useful imaging of the ear. J Radiol 2006;87:1728-42

4. Teszler CB, Daval M, Altabaa K, et al. Computed tomography-based workup of conducted hearing loss with normal middle ear: don't forget superior semicircular canal dehiscence. Int Tinnitus $J$ 2008;14:53-56

5. El Kohen A, Teszler CB, Williams MT, et al. Superior semicircular canal dehiscence mimicking otosclerosis: usefulness of imaging. Ann Otolaryngol Chir Cervicofac 2007;124:330-32

6. Prades JM, Veyret C, Saliou G, et al. Normal anatomy and imaging of human temporal bone. Rapport SFORL 2010:39-75

7. Chole RA, McKenna M. Pathophysiology of otosclerosis. Otol Neurotol 2001;22:249-57 
8. Shine NP, Rodrigues S, Miller S, et al. Bilateral stapedectomy: association between first- and second-ear surgical findings and their effects on the second-ear outcome. Ann Otol Rhinol Laryngol 2008; 117:207-11

9. Daniels RL, Krieger LW, Lippy WH. The other ear: findings and results in 1,800 bilateral stapedectomies. Otol Neurotol 2001;22:603-07

10. Inserra MM, Mason TP, Yoon PJ, et al. Partial promontory tech- nique in stapedotomy cases with narrow niche. Otol Neurotol 2004;25:443-46

11. Ayache D, Sleiman J, Tchuente AN, et al. Variations and incidents encountered during stapes surgery for otosclerosis. Ann Otolaryngol Chir Cervicofac 1999;116:8-14

12. Lippy WH, Battista RA, Berenholz L, et al. Twenty-year review of revision stapedectomy. Otol Neurotol 2003;24:560-66 